\title{
Retraction Note to: Structure-based development and optimization of therapy antibody drugs against $\mathrm{TNF} \alpha$
}

\author{
Wenyan $\mathrm{Fu}^{1,2,3} \cdot$ Xiaoze Wang $^{4,5} \cdot$ Weili Yang ${ }^{5}$ Hiroaki Takeda ${ }^{3,6} \cdot$ Shi Hu ${ }^{1,2}$. \\ Zhiyong Lou ${ }^{7} \cdot$ Jian Zhao ${ }^{1,2} \cdot$ Augus N. Bethune ${ }^{3,8} \cdot$ Yajun Guo $^{1,2,5}$
}

Published online: 9 December 2015

(C) Springer-Verlag Wien 2015

\section{Retraction to: Amino Acids (2015) 47:1259-1266 \\ DOI 10.1007/s00726-015-1954-9}

The Publisher and Editor retract this article in accordance with the recommendations of the Committee on Publication Ethics (COPE). After a thorough investigation we have strong reason to believe that the peer review process was compromised. In addition some author identities and given institutional addresses could not be confirmed.

The online version of the original article can be found under doi:10.1007/s00726-015-1954-9.

Augus N. Bethune

augus.bethune@aftertumor.com

$\triangle$ Yajun Guo

yjguo_smmu@163.com

1 Key Laboratory of Oncology, Cancer Center, Chinese PLA General Hospital and Chinese PLA Medical School, Beijing, People's Republic of China

2 Translational Medicine Research Institute and International Joint Cancer Institute, The Second Military Medical University, Shanghai, People's Republic of China

3 Tianjin Joint Academy of Biomedicine and Technology, Tianjin 300457, People's Republic of China

4 Graduate School of Medicine, Nagoya University, Showa-ku, Nagoya, Japan
5 School of Medicine, Nankai University, Tianjin, People's Republic of China

6 Department of Biomedicine, Nagoya University, Showa-ku, Nagoya, Japan

7 Laboratory of Structural Biology and MOE Laboratory of Protein Science, School of Medicine, Tsinghua University, Beijing, People's Republic of China

8 Molecular Drug Discovery Center, Xi' an Jinwa Pharmaceutical Co., Ltd, Xi' an 710000, Shaanxi, People's Republic of China 